\title{
Lobular Breast Carcinoma In Situ
}

National Cancer Institute

\section{Source}

National Cancer Institute. Lobular Breast Carcinoma In Situ. NCI Thesaurus. Code C4018.

A non-invasive adenocarcinoma of the breast characterized by a proliferation of monomorphic cells completely filling the lumina. The overall lobular architecture is preserved. It is frequently multifocal ( $90 \%$ in some series) and bilateral. It seldom becomes invasive; however there is an increased risk of infiltrating ductal adenocarcinoma. 